\section{Impacts of vehicle tire on slip resistance and sound pollution in asphalt pavements}

\author{
Saeed Abbassi ${ }^{*},{ }^{1}$, Muhammad Kazemi $^{1}$, Nima Norouzi $^{2}$, Zahra Nasiri ${ }^{3}$ \\ ${ }^{1}$ School of Civil Engineering, University of Zanjan, Zanjan, Iran. \\ ${ }^{2}$ Department of energy engineering and physics, Amirkabir university of technology (Tehran polytechnic), 424 Hafez Avenue, \\ PO Box 15875-4413, Tehran, Iran.
}

${ }^{3}$ Department of Architecture, Islamic Azad university: Ardabil Branch, Ardabil, Iran.

\section{Keywords}

\section{Texture,}

Paving,

Noise,

Vehicle tire,

asphalt pavements

\begin{abstract}
Noise pollution caused by vehicle traffic is one of the major problems in urban areas with road expansion. Due to the increase in the cost of construction and installation of sound walls to deal with noise pollution, to deal with this problem should look for methods that do not have additional costs and operating costs. Improving the pavement texture is one of the most effective ways to reduce tire noise and pavement and reduce the asphalt surface's sound. To evaluate the slip resistance of asphalt, the English pendulum test according to ASTM E303-74 standard was performed on wet parts of asphalt in wet conditions. This device is used to examine the fine texture of the pavement. The number of pavement friction with a negative coefficient of 0.1469 has an inverse ratio with the intensity of sound level increases the number of pavement friction aligned with the amount of sound level created decreases. On the other hand, the depth of pavement texture, which is determined as the size of pavement materials, with a coefficient of 0.2810 , has a direct ratio with the amount of noise pollution, and the smaller the number of fine-grained materials used will increase the sound level. According to the results of the coefficients estimated from the equation, it can be concluded that the preparation of pavements with an amount of friction can reduce the amount of noise pollution emitted by the movement of vehicles, especially in urban areas and sensitive areas. Therefore, it is recommended that in acoustically sensitive areas, in preparing pavements, arrangements be made to use coarser materials and maintain proper pavement resistance. For this purpose, in this article, the pavement's texture is examined in the amount of noise created due to the tire's interaction and the pavement.
\end{abstract}

\section{Introduction}

Vehicle control and aircraft landing safety depend to a large extent on pavement surface characteristics. If the pavement is dry, the friction between the tire and the pavement surface is usually high. In cold and stormy weather, the presence of water (humidity) increases the potential for hydroplaning or slipping and creates a critical situation, especially when the pavement surface resistance to slipping is low. On a wet pavement surface that does not have sufficient slip resistance and hydroplaning, the driver or pilot will not maintain direct control over the vehicle. The main reason for collecting information about slip resistance of pavement surfaces is to prevent accidents or reduce them. This data is used to identify parts of the pavement that have low or rapidly decreasing slip resistance. As a result, management can use this information as a tool to prioritize and select the appropriate repair and maintenance [1, 2].

Noise, vibration, and harshness (NVH), a.k.a., noise and vibration $(\mathrm{N} \& \mathrm{~V})$, represents a quality factor in automotive engineering [3]. It is generally accepted that there are five major sources of noise for a running vehicle: engine (power train), intake system, exhaust system, aerodynamic turbulence (wind), and tire-pavement interaction [4, 5] The power train noise is generated primarily due to combustion in the engine, gas flow, and the transmission parts' mechanical movement. The aerodynamic noise depends on vehicle geometry and speed of the vehicle, generated due to aerodynamic friction and turbulence caused by the vehicles moving at higher speeds. The combination of the engine (power train), intake system, the exhaust system is also called the propulsion system which the performance is largely dependent on the engine speed; they have coherent noise sources and are interacting with each other, but they are incoherent with tire noise [6]. It is reasonable to infer that lower gear generates more noise for the same vehicle speed since the engine speed is higher[7]. It can be seen that the noise level is relatively independent of torque except when rpm is below 1000. The intake orifice noise versus engine speed is displayed in [8]. The exhaust noise versus engine speed is displayed in [9], and its typical spectrum is shown in [5]. For vehicle noise from trucks, the supplementary braking noise (pneumatic cylinders) and "body slap" is often important [10].

Slip resistance is defined as the force that acts against the tires slipping on the pavement surface while preventing the tires from spinning. Although slip resistance is considered a feature of pavement surface, conditions other than pavement surface conditions affect the operating force between the tire and pavement: including tire pressure, tire tread, water presence, loading temperature, and vehicle speed. Vehicle hydroplaning occurs when the rubber surface and the pavement surface are separated by water or contaminants [2].

Reducing friction between the tire and pavement joint is one factor that causes and increases road accidents. The amount of friction depends on the road surface characteristics and the vehicle's characteristics, critical in wet road conditions. On the road with low slip resistance, more accidents than on the surface with slip resistance are high. About one-third of road accidents are caused by a vehicle slipping on a wet surface [3,4]. Friction is a force that always acts in the opposite direction of motion. The friction coefficient is defined as the frictional force's ratio acting on the joint plane and the force perpendicular to that plane. In physics, $\mu$, the friction coefficient is calculated by dividing the abrasion resistance due to 
motion at the joint surface, (F), by the load acting vertically (L). The pavement characteristic that affects the sliding resistance is the coarse and fine texture of the paved road. However, pavement engineers usually prefer to calculate the friction as the friction ratio $f=F / L$, where $\mathrm{F}$ is the friction force, and $\mathrm{L}$ is the vertical force[5].

Because it is not correct to attribute the f-property to the pavement without specifying the parameters that affect it, such as the wheel, speed, temperature, water-thin layer, etc., standards have been developed to measure slip resistance. The most popular standard is ASTM. According to this standard, the parameter measured is called the slip number (SN) and is calculated as equation (1):

$$
S N=100 \times f
$$

Slip resistance measurements are usually performed on wet pavements. The SN values obtained from a standard method indicate a pavement's ability to maintain the friction required by vehicles. In this case, by measuring the sliding resistance of the pavement in wet conditions for the vehicle, the amount of sound produced by the sliding resistance is evaluated, and the effect of sliding resistance with the sound produced by the tire on the pavement is obtained. All methods of measuring pavement strength are based on experience [1]

In an article entitled "The Performance of Porous Asphalts in Reducing Traffic Noise" (6), a very important issue about traffic noise is that the source of sound production, the vehicle, is itself the set of sources of sound production. Engine noise, air movement around the vehicle body, shifting, braking, horn, wheel movement on the street surface, and even music playback systems are all sources of sound in the vehicle. Efforts have been made to improve the streets and roads surface to reduce noise and vibration caused by cars' movement. Creating a porous structure in the asphalt pavement of road surfaces can reduce the emitted sound by absorbing and dissipating sound energy. In addition to reducing traffic noise, this porous structure also has advantages in the drainage of rainwater. Porous asphalt was first used in the United States in the 1950s. In the following years, its manufacturing technology in terms of granulation, space, strength, and stability increased. Despite its significant benefits in reducing noise, the use of these asphalts is also associated with problems. Retention of water droplets, dust, and sand in empty asphalt spaces, rapid aging of bitumen and rapid brittleness of asphalt, reduced efficiency of repaired asphalts, and the need for high technology for production are some of the cases that use this method to control traffic noise with He faces a problem. Therefore, due to the mentioned problems, porous asphalts' efficiency in reducing noise is not stable and decreases over time. However, their undeniable benefits have led to continued research on this technology as one of the most effective methods of reducing ambient noise. This article tries to express the latest studies and achievements in improving this technology's efficiency [6]. In an article [7], research has been done on tires' finite element modeling. A 155/65R13 tire is modeled on tire pressure, vehicle weight, and constant speed. In this study, the effect of the bel reinforcements' angle, the coefficient of friction between the tire and the pavement, and the ply materials are focused. One of the points mentioned in this article is the angle of the belt amplifiers in the tire, which greatly impact the model's resistance.

Considering the mentioned facts, this article is aimed to find a relationship between pavement parameters and its sound pollution. This equation can be used to calculate each parameter's optimum state to have the minimum sound pollution. The mentioned aim can fill the gaps of mentioned literature as they lack quantitative formulation of the pavement vs. sound pollution.

\section{Pavement texture}

The surface texture is defined as fine texture and coarse texture. The fine texture makes a grain look smooth or uneven when touched by hand. Figure (1) shows the pavement textures in the asphalt of the roads.

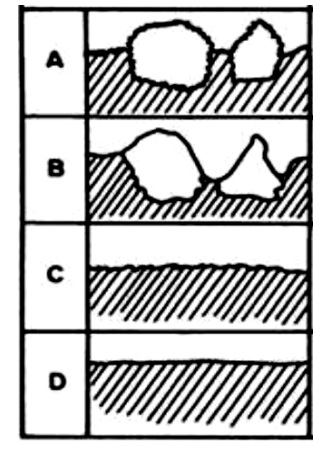

Figure 1. Terms used to describe the surface of road pavement [2].

The fine texture of the surface contributes to friction because it causes ballistic adhesion. The coarse texture is due to the shape, size, and arrangement of the grains (in flexible pavements) with the surface finish (in concrete pavements). Drainage is another important feature of the pavement surface. Water outlet ducts are provided in a proper drainage system, making contact between the tire and the pavement surface. The drainage system's effectiveness can be evaluated by measuring the friction ratio immediately after spraying water on the pavement surface and at regular intervals after determining the increase in friction [2].

In general, friction is a force that always acts in the opposite direction of motion, and the coefficient of friction is defined as the ratio of the horizontal reaction force in the direction of motion and the force perpendicular to this direction.

In other words, the coefficient of friction is equal to the kinetic resistance force (wear force between the tire and the pavement) divided by the vertical force. Higher coefficients of friction mean that more force is mobilized when resisting vehicle movement, and as a result, vehicle speed decreases and stops faster. The slip between the tire and the pavement surface affects the frictional resistance. The slip percentage according to STM E 1859 is according to equation (2) [8]:

$$
s=\frac{V-V_{R}}{V}
$$

In this equation:

\section{S: slip percentage}

V: Car's test Speed

$V_{R}$ : Tire rotation speed test

And so the slip velocity is according to equation (3)

$$
s=\frac{S \times V}{100}
$$

That :

s: slip speed

\section{S: Slip percentage [according to equation (2)]}

Therefore, when the locked wheel slides along the surface, the tire rotation speed is zero, and the tire has no rotation. In this case, the slip percentage is $100 \%$, and the slip speed will be equal to the test car's speed. In test equipment with low slip speed, the rotational speed $V_{R}$ will not be zero, and the slip percentage is less than 100 , and consequently, the slip speed is less than the speed of the test car.

Changes in the friction coefficient with the tire's slip velocity are shown in Figure (2). The slip velocity at which the friction coefficient reaches its maximum value is the critical slip velocity [5]. Then, due to the wheel's sliding speed, the coefficient of friction gradually decreases [8]. 


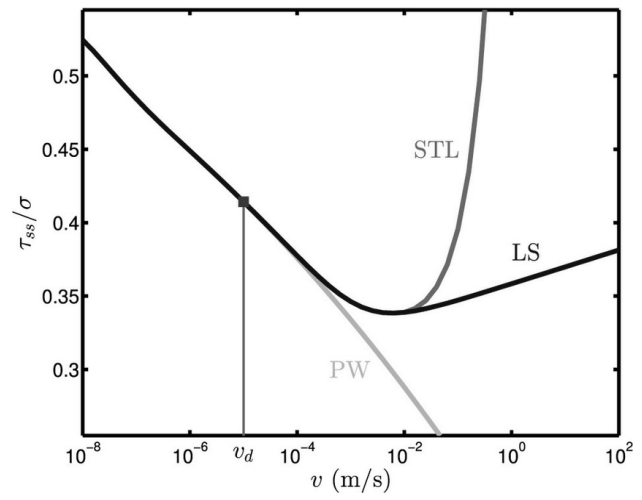

Figure 2. Curve measuring coefficient of friction vs. slip velocity[5].

According to Figure (2), it can be seen that in the area between zero slip velocity and critical slip velocity, the tire-related characteristics have the greatest effect on the slip-friction velocity curve. After the critical slip velocity (i.e., where the friction coefficient is maximum), the pavement surface characteristics have the greatest impact on the slip velocity-friction curve. Some friction measuring devices operate at low slip speeds. For this group of vehicles, tire specifications have an effect on the results obtained. Therefore, changes in tire properties can have significant effects on test results [8].

\section{Methodology}

The English pendulum test following ASTM E303-74 standard in wet conditions on different parts of ordinary asphalt in Tehran was used to evaluate porous asphalt's slip resistance. This device is used to examine the texture of the pavement[9]. The result of this experiment is reported as English pendulum numbers (BPN). The results are presented in Tables [1, 2], regarding the English pendulum test according to ASTM E-303 standard to measure the number of surface friction of asphalt pavement in the south to north routes of Valfajr town, north to south of Amirabad street and east to west of Fatemi street in Tehran. The sound is measured using a design vehicle, a Peugeot 206. In the quick setting mode, the sound level meter TES1357 and the height of 1.2 meters, and the distance of 7.5 meters from the vehicle's passing axis on both sides are measured. The test site's environmental and acoustic conditions, including road surface specifications, are selected following ISO 11819-1 [10]. In each section, five points with distances of 200 meters were selected, and a British pendulum machine measured the number of slippery asphalt pavement surfaces in the desired points

Table 1. The results of British pendulum number in asphalt pavement.

\begin{tabular}{lcc}
\hline Test location $(\mathrm{km})$ & Fraction & Number \\
\hline beginning & 69 & 3 \\
beginning & 64 & 2 \\
beginning & 63 & 1 \\
Middle & 65 & 3 \\
Middle & 66 & 2 \\
Middle & 66 & 1 \\
end & 67 & 3 \\
end & 63 & 2 \\
end & 61 & 1 \\
300 & 62 & 1 \\
350 & 63 & 1 \\
beginning & 64 & 1 \\
50 & 61 & 1 \\
\hline
\end{tabular}

According to equation (4), a relationship is established between BPN and $S N_{0}$ that can be used to calculate SNO [2].

$$
S N_{0}=1.32(B P N)-34.9
$$

wherein :
BPN: English pendulum number

$S N_{0}$ : number of slip starting from the origin,

According to equation (5), a relationship is established between MTD and PNG that can be used to calculate MTD [2].

$$
P N G=0.157(M T D)-0.47
$$

Wherein :

MTD: Pavement texture depth (inches)

PNG: Percentage of normal slope (mph)

According to equation (6), a relationship is established between V, PNG, SNO, and SN, which can be used to calculate MTD [2].

$$
\log (S N)=\log \left(S N_{0}\right)-0.00434(P N G) \times V
$$

wherein :

$\mathrm{V}$ : is the standard speed (40 $\mathrm{mph})$.

PNG: Percentage of normal slope (hours per mile)

$S N_{0}$ : slider number starting from the origin

SN: slip number

English pendulum numbers related to ordinary asphalt in Tehran BPN are placed in equation (4) to obtain $S N_{0}$ values. Given that this study's design speed is $50 \mathrm{~km} / \mathrm{h}$, the speed should be obtained in terms of miles per hour, equal to 31.25 miles per hour using interpolation and instead Consider the value 30. Using the values obtained for the variables V, SN, and $S N_{0}$ Placing them in equation (6), the value of the PNG variable is obtained, and since the value is in terms of hours per mile, it is multiplied by 0.63 to be converted to hours per kilometer. Using equation (5) and inserting the variable PNG value, the amount of pavement texture depth (MTD) is obtained. The resulting number, which is in inches, is multiplied by 25.4 to convert it to millimeters, and we put the values of the variables in the table (3-5). BPN has obtained in equation (4) $S N_{0}$ values; for these values in equation (6), negative PNG values are obtained. As a result, we omit these numbers, and also, the MTD values for the 50, 51 BPN numbers are obtained using interpolation. To evaluate the specimens' friction properties, the ASTM E303 standard was used under the heading "Standard Test Method for Assessing the Friction Properties of Procedures Using the English Pendulum Experimenter."The English pendulum machine is used to test on asphalt surfaces made in the laboratory and performed on-site and polished aggregate samples in convex molds to test the value of aggregate polishability (PSV). This device reduces energy when the slippery edge (Plastic shoes) goes on the sample, measures [11].

Table 3. The texture depth results in asphalt pavement (equation 46).

\begin{tabular}{cccc}
\hline MTD & PNG & SNO & BPN \\
\hline 0.100 & 1.5435 & 65.42 & 76 \\
0.200 & 1.4490 & 62.78 & 74 \\
2.300 & 0.4838 & 39.02 & 56 \\
0.300 & 1.1592 & 54.86 & 68 \\
0.900 & 0.7245 & 44.30 & 60 \\
1.100 & 0.6741 & 42.98 & 59 \\
3.200 & 0.3868 & 37.70 & 55 \\
9.400 & 0.0963 & 32.42 & 51 \\
0.900 & 0.7245 & 44.30 & 60 \\
0.700 & 0.7686 & 45.62 & 61 \\
10.90 & 0.0067 & 31.10 & 50 \\
9.400 & 0.0963 & 32.42 & 51 \\
\hline
\end{tabular}

Considering the three variables of noise level, slip resistance, and pavement texture depth, the relationship between these three variables can be obtained. To achieve this relationship, we use Minitab 
software. Because there is a high correlation between the two variables of slip resistance and pavement texture depth, linear regression can not be used, in which case the software output provides us with unstable coefficients. As a result, we use other proposed models to obtain a mathematical relationship between these three variables, and the proposed model is to use partial least squares.

Regression modeling to determine the relationship between sound level and friction number and pavement texture

To determine the relationship between sound level (as a dependent variable) due to the passage of vehicles with variables of pavement texture type (pavement material particles in millimeters) and pavement friction number (as independent variables), after determining the values of each variable, The above (experimenting) modeled it.

After collecting the data from the experiment, it was first checked that the obtained variables have the necessary conditions for ordinary least squares regression models [6]. Preliminary studies of the data showed that the collected independent variables (pavement texture depth and friction number) have a highly significant correlation (0.778) with each other. Therefore, one of the basic assumptions of regression models of ordinary least squares in which independent variables should have little or no alignment, and in this case, modeling with this method leads to unstable estimates of regression coefficients. Of course, alignment problems usually occur when the number of independent variables is very large or the available data is small. In the present study, considering that the collected data is small and there are only 12 observations, multiple alignments are not expected.

In such conditions, new usable modeling has been proposed, which can be called boundary regression [7], principal component regression [8], and partial least squares regression [9]. Among the mentioned models, the demarcation regression model is mostly used to diagnose multiple alignment problems and generally has no dependent variable prediction aspect. Usually, in such cases, one of the methods used is the partial least squares regression method. In this method, new variables are created to form the relationship between dependent variables and independent variables, which they call factors. Each of these factors is a linear combination of primary independent variables. Standard regression methods are then used to determine the equations that relate these factors to the dependent variable. Therefore, in the continuation of this research, using the partial least squares method to estimate the dependent variable of noise level with independent variables of pavement texture depth and friction number are given.

Partial least squares regression model for modeling the relationship between noise level and independent variables of pavement texture depth and friction number To regress modeling, one must first determine the independent and dependent variables. According to the experiments, three variables of noise level, pavement texture depth, and pavement friction number are available for modeling. What is clear is that the variables of pavement texture depth and friction number are exogenous. The noise level (in decibels) is an endogenous variable (i.e., two variables of pavement texture depth and friction number from outside affect the noise surface variable). Therefore, the dependent variable's noise level and the two variables of pavement texture depth and friction number constitute the independent variables of regression modeling (in this section, regression means partial least squares).

Considering that there are two independent variables in the present study, for the regression model with partial least squares, a factor was determined, and based on it, and the transfer function was obtained. All modeling calculations were performed in Minitab software, and then the cross-validation method was used to evaluate the validity of the obtained transfer function. In this way, data were excluded from the data set, and modeling was performed. The output data was then predicted according to the model obtained from other data. This process was repeated in the number of observations (i.e., 12 times). Also, the evaluation of the dependent variable's equation against the independent input variables was calculated by the indicators of good fit coefficient, good fit coefficient of prediction, and the sum of squares of predictive error [10], using Minitab software. It should be noted that the coefficient is a good fit coefficient of the transfer function obtained from the partial least squares regression, and the index is equal to the coefficient of determination between the measured values and the predicted values of the noise level in the mutual validation stage. It should be noted that the higher the sum and the fewer squares of the predicted error (PRESS), the higher the validity of the transfer function. The table of measured parameters, model analysis of variance table, model output, estimated coefficients of the transfer function, and good fitting coefficients are given in tables and graphs.

\section{Results and discussion}

Table (4) shows the characteristics of the measured parameters, number, minimum and maximum values, mean and standard deviation. $\mathrm{X}$ is the pavement texture depth in millimeters, $\mathrm{Y}$ is the dimensionless pavement friction number, and $Z$ is the noise level in decibels.

Table 4. The measured statistical parameters.

\begin{tabular}{ccccc}
\hline & Std.D & Mean & Max & Min \\
\hline $\mathrm{Y}$ & 4.09373 & 3.39420 & 10.19 & 0.190 \\
$\mathrm{X}$ & 8.63880 & 60.0833 & 76.00 & 50.00 \\
$\mathrm{Z}$ & 3.01063 & 74.5750 & 79.60 & 70.30
\end{tabular}

Table (5) shows the correlation values between the measured variables. As can be seen, there is a high correlation between the two variables of pavement texture depth and pavement friction number.

Table 5. The results correlations variables.

\begin{tabular}{cccc}
\hline $\mathrm{Z}$ & $\mathrm{X}$ & $\mathrm{Y}$ & \\
\hline $0.678(0.0115)$ & $-0.778(0.03)$ & 1 & $\mathrm{Y}$ \\
$-0.748(0.05)$ & 1 & & $\mathrm{X}$ \\
1 & & & $\mathrm{Z}$ \\
\hline
\end{tabular}

After examining the descriptive statistics of the measured parameters, partial least squares regression was modeled, and using this method, for independent variables of texture depth and pavement friction number, the transfer function was calculated by Minitab software. Equation (7) shows this function.

$$
Z=82.4494-0.1469 X+0.2810 Y
$$

Therefore, according to equation (7), the number of pavement friction with a negative coefficient of 0.1469 has an inverse ratio with the sound level; that is, by increasing the number of pavement friction, the amount of noise level decreases. Also, the depth of pavement texture, which is determined as the size of pavement materials, has a direct ratio with the amount of noise pollution with a coefficient of 0.2810 , and any size of finer materials will increase the sound level. Good statistical indicators of model fit are given in table (6). According to this table, a good fit coefficient is $57.44 \%$, which due to the small data and the number of low variables (two independent variables), a good fit has been created. Besides, a good prediction fit coefficient, a measure of the transfer function's validity, is close to $40 \%$, which is considered appropriate.

Table 6. The results of statistical parameters.

\begin{tabular}{lc}
\hline parameter & value \\
\hline fit coefficient & 0.5744 \\
prediction fit coefficient & 0.399 \\
Total squares of predictor error & 59.95 \\
\hline
\end{tabular}

A study entitled "Properties of pavement layer on tire noise from pavement" (13) concluded that different pavement materials could 
greatly impact the amount of sound emitted from it. In this study, a path with different materials has been constructed, and during two years, sound measurements have been performed on it in different conditions to determine the role of materials in reducing pavement noise. Finally, the volume of these levels is compared with each other. It is noteworthy that a youth level can reduce the volume, but it loses this property over time. The following results have been obtained according to this research:

- Pavement with impermeable surface texture increases the volume, so if we are looking to reduce the pavement's sound, we should reduce the surface texture as much as possible, but this will reduce road safety because it reduces the friction between the tire and the pavement.

- Porosity reduces the volume at all frequencies.

In a study conducted in this paper on pavement texture and volume, based on the results of a comparison of the residues given in Figure (3), it can be concluded that the variance of the residues against the fitted values is approximately the same, and the values The corrected residuals fluctuate around the fitted values around zero and do not follow a specific trend. On the other hand, these modified residues values do not have a specific trend against the order of observations. In addition to the above, the histogram and the probability diagram of the residuals can be seen in Figures (4 and 5).

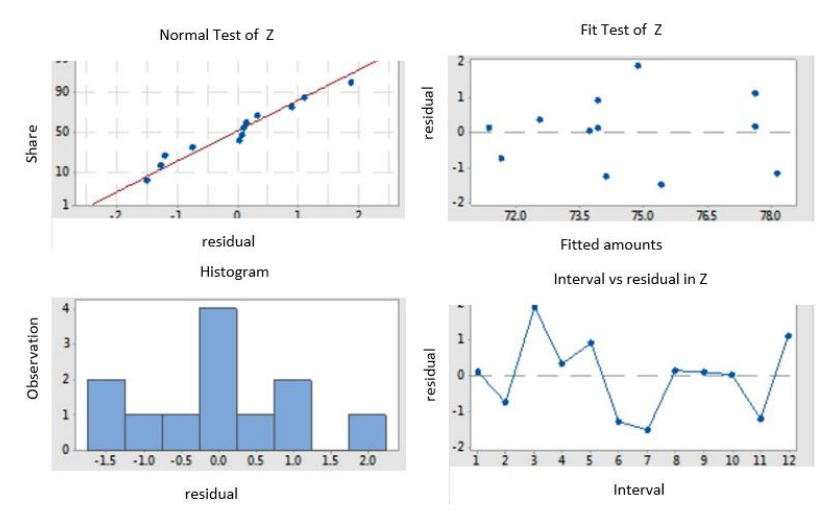

Figure 3. The results of residuals of modeling.

As mentioned in the modeling stage of this research, a crossvalidation method has been used to validate the model. The method of operation in this case of validation is that data is excluded from the data set and modeling is performed, then the output data is predicted according to the model obtained from other data. This process was repeated in the number of observations (12 times in this study). To see the validity of the paid model, Figure (4) is drawn. In this diagram, the dependent variable's real values (sound level) are on the horizontal axis, and the calculated values are on the vertical axis. The blue circles represent the fitted values, and the red squares represent the values obtained from the mutual validation. It is clear that the closer the two values are to each other, the better the model's validation.

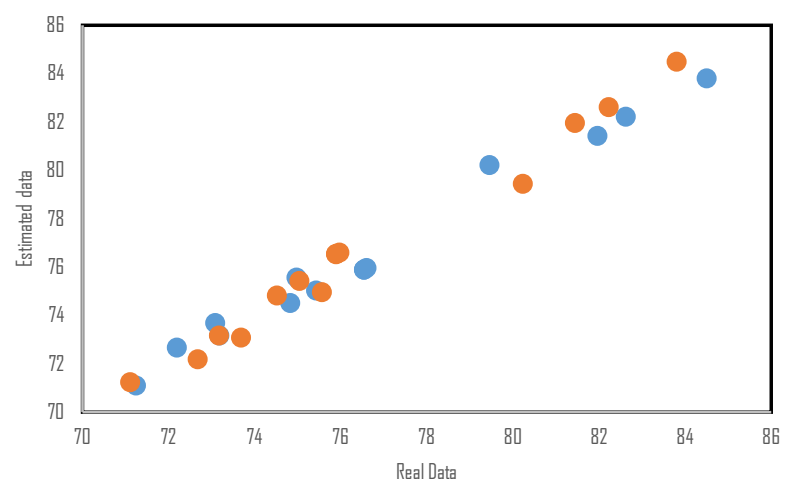

Figure 4. The results of validation parameters.

Leverage index has been used in this study to determine the presence of outliers among the collected data. Leverage measures the distance between the values of independent variables from their other values. Leverage values are usually plotted against standardized residual values to identify remote data.

Figure (5) shows the Leverages values versus the standardized residues. This diagram shows Leverages' values in the horizontal axis and standardized residues' values in the vertical axis. As can be seen, the standardized residual values are scattered around the Leverage value of 0.167 , and there is almost no observation (data) at a relatively large distance from this value in the data. Therefore, it can be concluded that outliers do not exist within the data obtained from the experiment and the model payment. Finally, Figure (6) shows the probability that the residues are normal with a 95\% confidence margin. As can be seen, all the rest are in this margin of confidence.

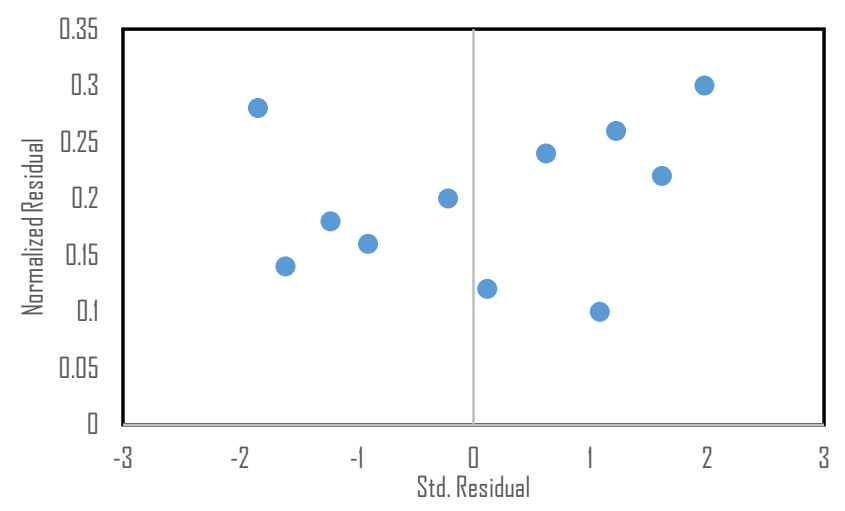

Figure 5. The results of residuals meaningless variables.

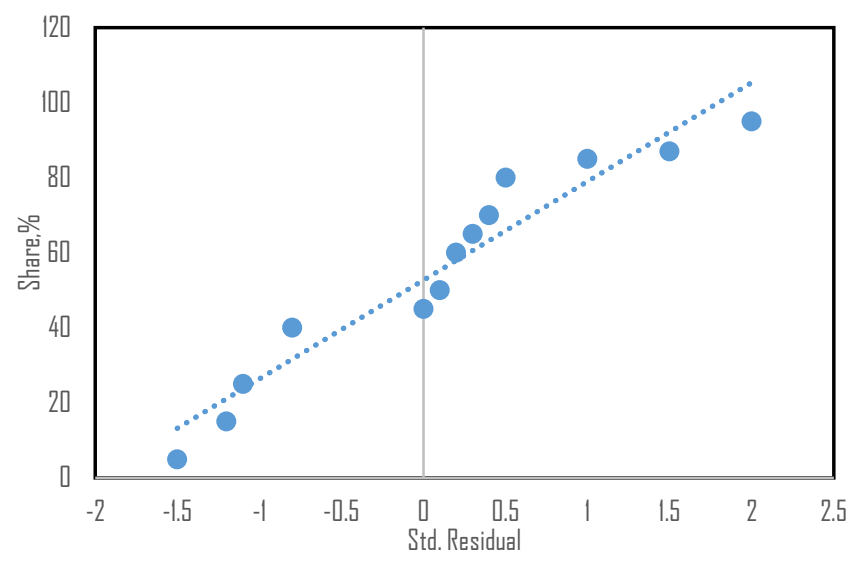

Figure 6. The results of residuals of variables with 95 percent confidence.

Therefore, according to equation (7), the number of pavement friction with a negative coefficient of 0.1469 has the inverse ratio of the noise level, i.e., increasing the number of pavement friction of noise level decreases. On the other hand, the type of pavement texture, which is determined as the size of pavement materials, has a direct ratio of 0.2810 with the noise level created, and any size of finer materials will increase the sound level. Using the results presented in the work of Haas et al. As well as in Henry's paper, the following results are obtained [1, 5]:

-Since reducing the depth of pavement texture reduces surface friction and may reduce safety, in studies related to the effect of pavement texture on sound production, safety should also be considered, and the balance should be sought. 
- The pavement age plays an important role in changing the sound level caused by the tire and pavement. Increasing the age of pavement increases the level of sound produced in asphalt pavements and decreases porous pavements.

- The quietest pavement for passenger cars is asphalt pavement with open granulation.

The contributions to noise reduction from tires and pavements cannot be considered separately. A "quiet tire" on one specific pavement may not be quiet on the other type of pavements; similarly, a "quiet pavement" may not work for all the tires, making sense because TPIN comes from the interaction between the tire and pavement. The noise performance cannot be determined if only the tire or pavement information is given. A study in 1998 reported that the crossplay truck tire (7.00R15) for medium trucks was among the noisiest over a relatively coarse chipseal pavement, but it was one of the quietest on a smoother chipseal pavement. A paper[1] investigated more than 2000 tire/road combinations and found that the sound reduction due to the combination of silent surfaces and silent tires was smaller than the numeric sum of both, especially for rough pavements, the silencing effects of quiet tires were marginal. A study [5] indicated that the Bridgestone B-250 had a relatively high type approval level (73 dBA) but was more silent on the Norwegian dense (and rough) surfaces than some other tires. The Michelin Energy Saver was rated as a quiet tire in terms of interior noise but was shown to be rather noisy in the exterior. However, Ref. [136] also demonstrated that some tires seemed to perform as low-noise tires independent of the type of pavements.

A paper [137] applied the Active Noise Control (ANC) for cabin noise from a power train $(40-500 \mathrm{~Hz})$ with advanced speakers. Sun et al (2012) [6] applied active noise control to the low-frequency structureborne vehicle interior noise from tire-road interaction and proved its efficiency. A study [4] investigated the active control of structureborne interior noise based on the separation of front and rear structural noise-related dynamics. However, the present author found no literature investigating the active noise control of exterior noise.

Due to the pressure from the regulations and customers, the tire/automotive companies and pavement organizations have endeavored to reduce the tire-pavement interaction noise. Tire industries attempted to optimize the tread pattern and tire construction for quieter tires, including pitch sequencing, tire cavity foam, tread, rim modifications, etc. Pavement industries attempted to modify the pavement texture and stiffness for quieter pavement. In the future, more and more research should be focused on the interaction between tire and pavement, which would lead to a quieter combination rather than quiet tire and quiet pavement separately. The challenge for both tire and pavement industries is that the acoustic performance of tire and pavement usually conflicts with other performances, such as traction, handling, rolling resistance, hydroplaning, and durability. Therefore, a compromise between noise reduction and maintaining other performances should be carefully considered and designed.

\section{Conclusion}

According to the results of the coefficients estimated from the above equations, it can be concluded that the preparation of pavements with a high amount of friction can reduce the noise level caused by the movement of vehicles, especially in urban and sensitive areas. Therefore, it is recommended that an arrangement be made to maintain proper pavement resistance; coarser grain materials should be used while preparing pavements in areas sensitive to the noise level. Of course, the relationship mentioned above can help researchers find the best possible state in which the sound level is minimized. For this purpose, it is suggested that the minimum and maximum allowable and standard values of pavement number and grain size be extracted according to the regulations, and the optima condition to have the lowest amount of noise pollution be calculated. Briefly, the presence of sound is inevitable, and the sound produced by the tire and pavement cannot be eliminated, but it may be possible to reduce this noise by considering parameters such as pavement texture.

\section{Nomenclature}

S : Percent slip

$\mathrm{V} \quad$ : Car's test Speed

$V_{R}$ : Tire rotation speed test

$\mathrm{S} \quad$ : slip speed

$\mathrm{S} \quad$ : Slip percentage [according to equation (2)]

BPN : English pendulum number

$S N_{0} \quad$ : number of slip starting from the origin,

MTD : Pavement texture depth (inches)

$\mathrm{V} \quad$ : is the standard speed (40 $\mathrm{mph}$ )

PNG : Percentage of normal slope (hours per mile)

SN : slip number

\section{Declaration of Conflict of Interests}

The author declares that there is no conflict of interest. They have no known competing financial interests or personal relationships that could have influenced the work reported in this paper.

\section{References}

[1.] Henry, J.J, Evaluation of Pavement Friction Characteristics. NCHRP Synthesis 291. Transportation Research Board. Washington DC, (2000).

[2.] Huang, Y. H., Pavement Analysis and Design, 2nd ed., University of Kentucky, second edition, (2004) 256- 259.

[3.] Jamieson, N., Dravitzki, V., 2006. Management of skid resistance under icy conditions on New Zealand roads, Land Transport New Zealand Research report 293 (2006) 68.

[4.] Jeffrey S. Kuttesch, Quantifying the relationship between skid resistance and wet weather accidents of Virginia data, blachsburg, Virginia, (2004) 154-165.

[5.] Haas, R., W.R. Hudson, and J.P. Zaniewski, Modern Pavement Management, Krieger Press, Malamar, Florida, (1994) 115- 126.

[6.] Jalili, sh, Sanati Fard, S, Porous Asphalt, 11th conference of civil engineers and students, Hormozgan University, (2004).

[7.] Malekzadeh, M., Rahimi, H, A Parametric Study on the Steady State Rolling Behaviour of a Steel-belted Radial Tyre, Iranian Polymer Journal 3 (2007) 56-58.

[8.] Gerardo W. Flintsch Kevin K McGhee, The Little Book of Tire Pavement Friction, Version 1.0, Submitted for Review and Comment Pavement Surface Properties Consortium (2012).

[9.] Ameri, M, Afandi zadeh, Sh, Miraee Moghadam, M.H, 2009, The methods for measuring skid resistance in the road surface, 11th conference of civil engineer $s$ and students, Hormozgan University (2009).

[10.] International standard ISO 11819-1. 'ERC 2004 'Methods for evaluation of road noise, pp 21-28.

[11.] Asi. IM, Evaluating Skid Resistance of Different Asphalt Concrete Mix, Building and Environment, 42 (2007) 325-329.

[12.] American Society for Testing and Materials, 2000.Test Method for Measuring Frictional Properties Using the British Pendulum tester, Philadelphia, USA, ASTM, E 303 - 93.

[13.] Sakhaeifar, M., Heitzman, M, The effects of pavement surface characteristics on tire/pavement noise, Landscape, and Urban Planning, (2014) 1300-1305.

\section{How to Cite This Article}

Abbassi, S., Kazemi, M., Norouzi, N., and Nasiri, Z., The effect of moving vehicle tires on slip resistance and sound production in asphalt pavements, Brilliant Engineering, 3(2021), 16-21. https://doi.org/10.36937/ben.2021.002.004 